\title{
Perspective taking and theory of mind in hide and seek
}

\author{
Chris N. H. Street ${ }^{1,2} \cdot$ Walter F. Bischof ${ }^{2,3}$ - Alan Kingstone ${ }^{2}$
}

Published online: 13 November 2017

(C) The Psychonomic Society, Inc. 2017

\begin{abstract}
Does theory of mind play a significant role in where people choose to hide an item or where they search for an item that has been hidden? Adapting Anderson's "Hide-Find Paradigm" Anderson et al. (Action, Perception and Performance, 76, 907-913, 2014) participants viewed homogenous or popout visual arrays on a touchscreen table. Their task was to indicate where in the array they would hide an item, or to search for an item that had been hidden, by either a friend or a foe. Critically, participants believed that their sitting location at the touchtable was the same as - or opposite to - their partner's location. Replicating Anderson et al., participants tended to (1) select items nearer to themselves on homogenous displays, and this bias was stronger for a friend than foe; and (2) select popout items, and again, more for a friend than foe. These biases were observed only when participants believed that they shared the same physical perspective as their partner. Collectively, the data indicate that theory of mind plays a significant role in hiding and finding, and demonstrate that the hide-find paradigm is a powerful tool for investigating theory of mind in adults.
\end{abstract}

Keywords Visual search · Embodied perception

Chris N. H. Street

c.street@hud.ac.uk

Alan Kingstone

alan.kingstone@ubc.ca

1 Department of Psychology, University of Huddersfield, Huddersfield HD1 3DH, UK

2 Department of Psychology, University of British Columbia, Vancouver, BC V6T 1Z4, Canada

3 Department of Computing Science, University of Alberta, Alberta T6G 2E8, Canada
Studies of visual search normally involve participants looking for an item that is presented in clear view but made difficult by the presence of visually similar distractors (e.g., 'Where's Waldo?'), yielding important insights regarding human selective attention (Nakayama \& Martini, 2011; Wolfe, 2014). There is another type of search that has been left relatively untouched by researchers, although it is prevalent in everyday life. This form of search involves looking for something that is not in clear view, e.g., a set of keys or, in a far more serious situation, an explosive device concealed in a building (Legge et al., 2012; Peskin \& Ardino, 2003; Smilek et al., 2009; Talbot et al., 2009). The small handful of studies that have investigated this type of search have found that looking for a concealed item is not always well predicted by the principles derived from standard search experiments (Anderson et al., 2014; Foulsham et al., 2014).

The present study explores a particular instance of this new line of work, specifically, how hiding and finding a target item is carried out when concealed by either a friend or a foe. The introduction of a social context - friend or foe-is especially noteworthy because it has been proposed that such a situation engages a participant's theory of mind (Anderson et al., 2014). For instance, when participants perceived that their partner was a friend rather than a foe, they were more inclined to (1) select nearby locations on homogenous displays and (2) select the visually unique item on popout displays. This was true for both hiding and finding. Anderson et al. (2014) interpreted these findings as indicating that participants intuit that a friend, rather than a foe, would hide or search for a location that was easy to reach or that captured attention. That is, participants "simulated" (Flavell, 2004) what would be easy and attention-grabbing when considering where to hide/find an item involving a friend or foe.

Theory of mind involves adopting another person's perspective in order to consider their beliefs and intentions (BarnesHolmes et al., 2004; Flavell, 2004; Vogeley et al., 2001). 
Anderson et al. (2014) implicitly assumed that participants systematically began with the belief that their partner would be sitting on the same side of the table as themselves. This confounds a participant's position with the partner's assumed position, and so one could reasonably argue that participants had no need to adopt a theory of mind regarding their partner's perspective because the participants could rely on their own perspective.

The present study tackles this issue by manipulating whether participants think that they are sitting in the same location as a friend or foe, or on the opposite side of the table. Specifically, the task is to either (1) hide an item from a friend or foe, or (2) find an item hidden by a friend or foe. Two classic categories of visual displays were used: popout displays (one item carries a unique feature) and homogeneous displays (all items are the same). This enabled us to vary the complexity of the visual world within which hiding and finding would occur in a manner that converged with well-established search environments. These manipulations of task type and display follow those of Anderson et al. (2014), with the key exception being that the location of the friend or foe was either on the same or opposite side of the table as the participant.

Foundational to a theory of mind account is that people can think about someone else's mind by moving beyond their own perspective (see Barnes-Holmes et al., 2004; Vogeley et al., 2001). This predicts that participants will be sensitive to a shift in a partner's perspective even when it differs from their own location, and this will systematically impact their decision and behavior. For instance, participants might decide to hide an item that they intend their partner to find by placing it near to their partner - even if that means reaching to the far opposite side of the table to place it near to the partner's position. The null hypothesis is that people perform the hide-find task by determining what they would do, as a friend or foe, based on their own perspective. As such it predicts that participants will be insensitive to their partner's perspective, for example, tending to place an item near to themselves when making it easy to find, even if the partner is sitting far away on the opposite side of the table.

\section{Methods}

\section{Participants}

Seventy-six participants $\left(\mathrm{M}_{\mathrm{age}}=20.26\right.$ years, $\mathrm{SD}_{\mathrm{age}}=2.42$, range 17-31 years), of which 51 were female, took part for course credit or $\$ 10 \mathrm{CAD}$. They were randomly assigned to four groups of equal size, with each group possessing a unique combination of hide/find and same/opposite table sides, e.g., Group 1 as Hide Condition/Same tableside. ${ }^{1}$

\footnotetext{
${ }^{1}$ Given the sample size, power of $0.90, \alpha$ of 0.05 , and an assumed correlation between samples of 0.50 , an ANOVA has sufficient power to detect effect sizes as small as $\eta_{\mathrm{p}}{ }^{2}$ of 0.07 (sensitivity analysis conducted using $\mathrm{G}^{*}$ Power 3.1: Faul, Erdfelder, Buchner, \& Lang, 2009).
}

\section{Materials}

A horizontal $93.9 \times 53.1 \mathrm{~cm}$ touchscreen was laid flat to act as a touch table. A virtual start bar was presented along the length of the screen nearest the participant. Sixteen squares were arranged in a square grid above the start bar, with each square $90 \times 90 \mathrm{~mm}$ in size and a $23 \mathrm{~mm}$ gap between each adjacent square (see Fig. 1). All participants could reach all cells easily.

On any given trial either (1) all 16 squares had an identical symbol drawn on them (a blue or green line drawn horizontally or vertically) called a homogenous display (96 trials total), or (2) 1 out of 16 items was selected randomly to have a feature that differed from the others in orientation (e.g., a vertical green line amongst horizontal green lines) or color (e.g., a horizontal blue line amongst horizontal green lines) called a popout display ( 96 trials total for each of the four rows). In total, there were 480 trials randomized by condition, symbol, and popout location.

\section{Procedure}

There was only one chair positioned at the table and it was always placed in the same location for all participants. If a participant was assigned to the "opposite condition", the chair was placed on the other side of the table before s/he arrived, and then the chair was repositioned under the observation of the participant. This bit of theatre was adopted to reinforce the manipulation that the participant was partnered with a person who was positioned on the opposite side of the table. If the participant was in the "same condition" the chair was not moved before $\mathrm{s} / \mathrm{he}$ arrived. In each case, the participant sat in the same seating position regardless of condition.

Once the participant was seated, the screen presented instructions to touch the near or far side of the table to rotate the screen image and instructions. This additional theater supports further the idea that the participant was partnered with a person who was positioned on the opposite or same side of the table. Next the participant read instructions (see Table 1). The instructions were also explained verbally by the experimenter, and participants were invited to ask questions if they needed any points clarified.

At the start of each trial (see Fig. 1), participants could see the start bar with the word FRIEND or FOE written on it, plus 16 empty squares. Participants held their finger down on the start bar. After a random duration between $1 \mathrm{~s}$ and $2 \mathrm{~s}$, the symbols appeared on the squares and the friend/foe word disappeared. At this point a participant could select where they would hide or find an item. If participants released the start bar too early, the words TOO EARLY were printed on the start bar and participants had to wait $1 \mathrm{~s}$ before the trial began again.

Twelve blocks of 40 trials composed a testing session. In the first six blocks, participants were told the partner was either a friend or a foe. In the last six blocks they were told 


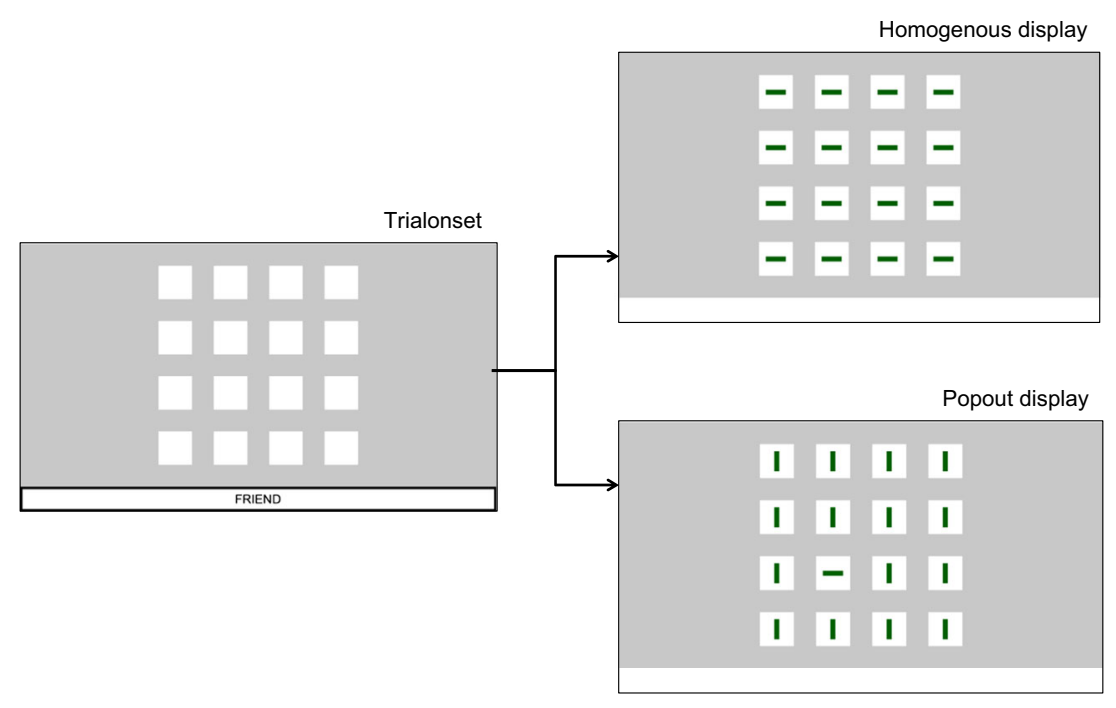

Fig. 1 A trial began with the word FRIEND or FOE appearing on the start bar, instructing the participants to consider their partner as a friend or a foe. A homogenous or popout display [unique feature orientation (as

the partner was a foe or friend, respectively. The order was counterbalanced between participants. Between each block the instructions were presented again, thereby providing participants with the opportunity to take a break if they wished.

\section{Design}

The between-subject manipulations were task type (hide or find) and tableside position (partner on the same or opposite tableside). The within-subject manipulation was relationship (friend or foe) and visual display (homogenous or popout). The primary dependent variable was which square was selected on each trial. We also measured decision time, but it was not emphasized to participants to respond as quickly and accurately as possible, and it did not yield any significant effects save for one exception noted below.

\section{Results}

To summarize the findings reported below, they indicate a role both for beliefs about other people's intentions in hiding and finding behaviors as well as their affordances for action. There is a bias towards selecting items that are physically closer to oneself, but this bias is modulated by whether the partner is thought to be a friend or foe and whether that person is believed to be viewing it from the same side or the opposite side of the table. Our findings also indicate differences in hiding versus finding behaviors, suggesting again that different forms of information or different processes underlying them. Unreported effects were statistically non-significant at $\alpha=.05$. shown) or color] was then presented. Note the popout feature could be a unique orientation (as shown here) or color. Participants touched one of the 16 squares to indicate their hide or find decision

\section{Homogenous trials}

Anderson et al. (2014) found a strong tendency for participants to choose items in the two rows nearest themselves on homogenous displays. But the extent of this near bias was dependent on participants' beliefs about the partner. The proportion of these near responses are considered in detail below ${ }^{2}$.

A 2 (friend/foe, within-subject) $\times 2$ (same/opposite tableside, within-subject) $\times 2$ (hide/find, between-subject) mixed ANOVA was conducted on the proportion of near responses. There was a main effect of relationship, $F(1,81)=5.32, P=.024, \eta_{\mathrm{p}}{ }^{2}=0.06$, such that participants showed a greater near bias when the partner was a friend $(M=.71, \mathrm{SD}=.28,95 \% \mathrm{CI}[.64, .77])$ than a foe $(M=.62, \mathrm{SD}=.22,[.57, .66])$. In addition, a relationship by tableside interaction was returned, $F(1,81)=14.56, P<.001, \eta_{\mathrm{p}}{ }^{2}$ $=0.15$, as illustrated in Fig. 2 . Bonferroni-corrected $t$-tests found a greater near bias when the partner who sat on the same (near) tableside of the screen was a friend rather than a foe, $t(81)=4.23$, $P<.001, \mathrm{~d}_{\mathrm{z}}=0.47$. When participants believed that the partner was positioned on the opposite side of the table, there was no evidence of an effect of seating position, $t(81)=-1.09, P=.272$, $\mathrm{d}_{\mathrm{z}}=-0.12$. This variation in the near bias as a function of the relationship (friend/foe) and sitting position (same/opposite tableside) is consistent with the theory of mind position. Finally, there were no main effects or interactions with the task type hence the figure shows the combined results.

Decision time was also shorter for friends $(M=1001 \mathrm{~ms}$, $\mathrm{SD}=226 \mathrm{~ms}, 95 \% \mathrm{CI}[952,1049])$ than for foes $(M=1206$ $\mathrm{ms}, \mathrm{SD}=580 \mathrm{~ms},[1079,1333])$. There were no main effects of tableside position or task type (hide/find), all $F_{\mathrm{s}}<1$.

\footnotetext{
${ }^{2}$ A heatmap of responses for all cells reveals that responses decline as row distance increases. In the nearest row, slightly more responses are made on the right, reflecting an effect of handedness.
} 
Table 1 Instructions split by task type, partner type, and partner position

Instruction

\begin{tabular}{|c|c|c|c|}
\hline & & \\
\hline & & Same side of table & Other side of table \\
\hline \multirow[t]{8}{*}{ HIDE } & \multirow[t]{4}{*}{ Friend } & $\begin{array}{l}\text { Hide an object under one of the squares } \\
\text { so someone WILL find it }\end{array}$ & $\begin{array}{l}\text { Hide an object under one of the squares so someone } \\
\text { WILL find it }\end{array}$ \\
\hline & & $\begin{array}{l}\text { Much like you might hide a spare key } \\
\text { outside your home so someone can find it easily }\end{array}$ & $\begin{array}{l}\text { Much like you might hide a spare key outside } \\
\text { your home so someone can find it easily }\end{array}$ \\
\hline & & $\begin{array}{l}\text { The other participant looking for the object will view } \\
\text { the screen from the same side of the table as you }\end{array}$ & $\begin{array}{l}\text { The other participant looking for the object will view } \\
\text { the screen from the other side of the table from you }\end{array}$ \\
\hline & & Touch the square where you would hide the item & Touch the square where you would hide the item \\
\hline & \multirow[t]{4}{*}{ Foe } & $\begin{array}{l}\text { Hide an object under one of the squares so someone } \\
\text { WILL NOT find it }\end{array}$ & $\begin{array}{l}\text { Hide an object under one of the squares so someone } \\
\text { WILL NOT find it }\end{array}$ \\
\hline & & $\begin{array}{l}\text { Much like you might hide a spare key outside } \\
\text { you home so no one can find it easily }\end{array}$ & $\begin{array}{l}\text { Much like you might hide a spare key outside your home } \\
\text { so no one can find it easily }\end{array}$ \\
\hline & & $\begin{array}{l}\text { The other participant looking for the object } \\
\text { will view the screen from the same side } \\
\text { of the table as you }\end{array}$ & $\begin{array}{l}\text { The other participant looking for the object will view } \\
\text { the screen from the other side of the table from you }\end{array}$ \\
\hline & & Touch the square where you would hide the item & Touch the square where you would hide the item \\
\hline \multirow[t]{8}{*}{ FIND } & \multirow[t]{4}{*}{ Friend } & $\begin{array}{l}\text { An object was hidden under one of the squares } \\
\text { so someone WILL find it }\end{array}$ & $\begin{array}{l}\text { An object was hidden under one of the squares so someone } \\
\text { WILL find it }\end{array}$ \\
\hline & & $\begin{array}{l}\text { Much like you might hide a spare key outside } \\
\text { your home so someone can find it easily }\end{array}$ & $\begin{array}{l}\text { Much like you might hide a spare key outside your home } \\
\text { so someone can find it easily }\end{array}$ \\
\hline & & $\begin{array}{l}\text { The other participant hiding the object viewed } \\
\text { the screen from the same side of the table as you }\end{array}$ & $\begin{array}{l}\text { The other participant hiding the object viewed the screen } \\
\text { from the other side of the table from you }\end{array}$ \\
\hline & & Touch the square where you would find the item & Touch the square where you would find the item \\
\hline & \multirow[t]{4}{*}{ Foe } & $\begin{array}{l}\text { An object was hidden under one of the squares } \\
\text { so someone WILL NOT find it }\end{array}$ & $\begin{array}{l}\text { An object was hidden under one of the squares so someone } \\
\text { WILL NOT find it }\end{array}$ \\
\hline & & $\begin{array}{l}\text { Much like you might hide a spare key outside } \\
\text { your home so no one can find it easily }\end{array}$ & $\begin{array}{l}\text { Much like you might hide a spare key outside your home } \\
\text { so no one can find it easily }\end{array}$ \\
\hline & & $\begin{array}{l}\text { The other participant hiding the object viewed } \\
\text { the screen from the same side of the table as you }\end{array}$ & $\begin{array}{l}\text { The other participant hiding the object viewed the screen } \\
\text { from the other side of the table from you }\end{array}$ \\
\hline & & Touch the square where you would find the item & Touch the square where you would find the item \\
\hline
\end{tabular}

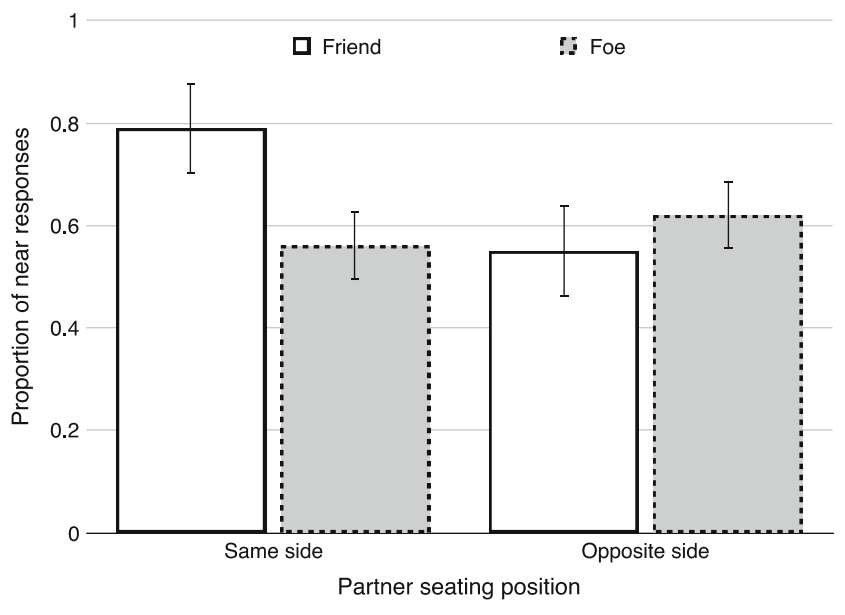

Fig. 2 Collapsed across task type, the degree of near bias ( $y$-axis) on homogenous trials as a function of a participant's belief about their partner's relationship (friend or foe, solid or dashed outline) and tableside position (same as, or opposite, the participant). Error bars represent $95 \%$ confidence intervals (CI)

\section{Popout trials}

We conducted a 2 (friend/foe) $\times 2$ (same/opposite $) \times 2$ (hide/find) mixed ANOVA on the proportion of trials where the popout item was selected. We found a main effect of relationship, $F(1,81)=117.50, P<.001, \eta_{\mathrm{p}}{ }^{2}=0.59$, with participants being more likely to select a popout item when the partner was thought to be a friend $(M=.64, \mathrm{SD}=.38,95 \% \mathrm{CI}[.55, .71])$ than a foe $(M=.14, \mathrm{SD}=.23,[.09, .19])$. This did not interact with the tableside position, $F(1,81)=0.04, P=.848, \eta_{\mathrm{p}}{ }^{2}<0.01$.

There was also a main effect of task type, $F(1,81)=4.94, P$ $=.029, \eta_{\mathrm{p}}{ }^{2}=0.06$. Participants were more likely to select the popout item when seeking the item $(M=.44, \mathrm{SD}=.32,95 \%$ CI $[38, .51])$ than when hiding it $(\mathrm{M}=.33, \mathrm{SD}=.34,[.26$, $.41]$ ). This bias to select the popout item during search did not interact with any other factor, all $F_{\mathrm{S}}<1$.

Finally, and novel to the present study, we examined where people chose to hide or find an item when not selecting the 
popout item. To address this question, we measured the distance from the popout item to the item that was selected, which could range from -3 (a selection distance of three rows from the popout towards the far (opposite) tableside) to +3 (a selection distance of three rows from the popout towards the near (same) tableside). We found that there was a tendency for participants to select items nearer to themselves relative to the popout item, resulting in positive mean directional distances.

A 2 (friend/foe $) \times 2$ (same/opposite $) \times 2$ (hide/find $)$ mixed ANOVA returned a main effect of relationship, $F(1$, 78) $=8.05, P=.006, \eta_{\mathrm{p}}^{2}=0.09$ : replicating the near bias findings found in homogenous trials, participants selected rows nearer to themselves when the partner was thought to be a friend $(M=.53, \mathrm{SD}=.73,95 \%$ CI $[.37, .70])$ than a foe $(M=.24, \mathrm{SD}=.54,[.12, .37])$. And as with homogenous trials, this effect of friend versus foe interacted with tableside position (see Fig. 3), $F(1,78)=6.22, P=.015, \eta_{\mathrm{p}}{ }^{2}=0.07$. When the partner was thought to be on the same side of the table, participants selected items nearer to themselves for friend $(M=.66, \mathrm{SD}=1.05,95 \% \mathrm{CI}[.43, .90])$ than foe $(M=.12, \mathrm{SD}=.77,[-.06, .30]),[t(78)=3.71, P<.001, d=$ $0.59]$ trials. However, there was no evidence of a difference in directional distance between friend $(M=.40, \mathrm{SD}=1.01$, $[.17, .63])$ and foe $(M=.36, \mathrm{SD}=.75,[.20, .53])$ trials when the partner was thought to be on the opposite side of the table, $t(78)=0.25, P=.806, \mathrm{~d}_{\mathrm{z}}=0.05$.

\section{Discussion}

Does concealing an item, or searching for it, involve theory of mind? The present study demonstrates that hiding and finding is dependent both on the perceived intentions and the

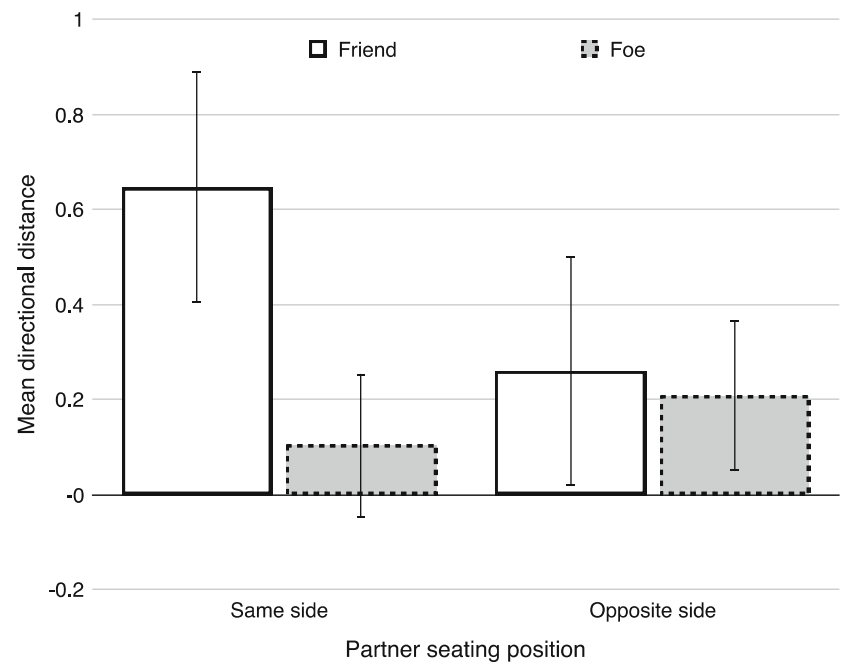

Fig. 3 Collapsed across task type, the directional distance as a function of a participant's belief about their partner's relationship (friend or foe, solid or dashed outline) and tableside position (same as, or opposite, the participant). Error bars represent $95 \%$ CI affordances of others. And critically, a theory of mind is employed, whereby people adopt another's perspective of how they might behave, rather than persisting with their own singular perspective.

There were three main findings recorded when participants believed that they were sitting on the same side as their partner. On homogenous displays, and for both hiding and finding, participants select nearby items more when partnered with friends than foes. For popout displays, and again for both hiding and finding, participants select the popout item when partnered with friends, and avoid the popout item when partnered with a foe. Finally, we found that when participants do not select the popout item, they tend to choose items nearer to themselves when partnered with friends, and further from the themselves when partnered with a foe. In short, participants behaved in a manner consistent with a theory of mind, searching for and hiding items in conspicuous and easy-toreach locations when working with friends, and the opposite when working with foes. Furthermore, these data replicate those of Anderson et al. (2014), demonstrating the robustness of the effects.

Critically, when participants believed that they were sitting on the opposite side of the table as their partner, a very different set of results emerged. Specifically, the effect of distance (near and far) was modulated, and often abolished. One can immediately see why factoring in a partner's perspective on the opposite side of the table would tend to negate the robust results observed for the same side of the table. A key aspect of the findings of Anderson et al. (2014) is that the selection of an item that is near to, or far from, the participant is also near to, or far from, the partner. However, when the partner is on the opposite side of the table, what is near to the participant is far from the partner, and vice versa. Crucially, ambiguity arises from factoring in a partner's perspective that differs from one's own. In other words, participants appear to be engaging in a second-order theory of mind: 'I believe that you believe that...' (see Hedden \& Zhang, 2002; Hiatt \& Trafton, 2015).

Given our finding that theory of mind in adults can be studied via a deceptively simple hide-find paradigm opens a range of research questions for future investigation. First, what is the relationship between hiding and finding? We, like others before us (Smilek et al., 2009), found that the principles of visual search often overlap with the principles of hiding, although the two can diverge. For example, in the present study, we found again that there was a greater tendency to choose a popout item when searching for an item hidden by a friend than when hiding an item for a friend. This suggests that attentional capture by a unique singleton has a greater effect on search than concealment. Second, what role does learning play in hiding and finding? To date, we have avoided providing participants with feedback as to whether their decisions were successful or not, as such feedback quickly made the situation more complex. However, in real life one receives 
feedback on these decisions, and learns from them, e.g., in developing a schema of the type of behavior one's partner engages in. How this might impact hiding and finding, and to what extent it differs between the two, is very much an open question. Third, we have found evidence that item saliency and physical distance are two important variables that hiders and finders take into account, suggesting that processes relating to attention and embodied cognition are in play. But what about the individual items themselves? Will someone wishing to make an item hard to find conceal the item under an ugly or heavy item that searchers might be discouraged from exploring, or does a searcher intuit that this is precisely the place where a hider might place a target? One can readily imagine that in such situations, environment (Legge et al., 2012) and expertise (e.g., Carbone et al., 2007) will play a critical role (e.g., police searching a house for drugs, a border guard looking for a concealed weapon, or firefighters searching a smoke-filled building for collapsed people). Understanding the processes of this sophisticated set of dynamics is a largely untouched line of investigation.

In sum, the present study has demonstrated that the hidefind paradigm engages theory of mind. In doing so, it lends support for the notion that the hide and find paradigm offers an effective tool for engaging and manipulating mentalizing operations in adults. Accordingly, a broad range of new avenues for future investigation avail themselves. In short, while the paradigm is deceptively simple, the potential for complex applications beyond the laboratory is encouraging.

\section{References}

Anderson, G. M., Foulsham, T., Nasiopoulos, E., Chapman, C. S., \& Kingstone, A. F. (2014). Hide and seek: The theory of mind of visual concealment and search. Action, Perception and Performance, 76, 907-913.

Barnes-Holmes, Y., McHugh, L., \& Barnes-Holmes, D. (2004). Perspective-taking and theory of mind: A relational frame account. The Behavior Analyst Today, 5, 45-71.
Carbone, A., Ciacelli, D., Finzi, A., \& Pirri, F. (2007). Autonomous attentive exploration in search and rescue scenarios. In L. Paletta \& E. Rome (Eds.)., 4th International Workshop on Attention in Cognitive Systems (pp. 431-446). Springer: Heidelberg.

Faul, F., Erdfelder, E., Buchner, A., \& Lang, A. G. (2009). Statistical power analyses using $\mathrm{G}^{*}$ Power 3.1: Tests for correlation and regression analyses. Behavior Research Methods, 41(4), 1149-1160. https://doi.org/10.3758/BRM.41.4.1149

Flavell, M. P. (2004). Theory-of-mind development: Retrospect and prospect. Merill-Palmer Quarterly, 50(3), 274-290.

Foulsham, T., Chapman, C., Nasiopoulous, E., \& Kingstone, A. (2014). Top-down and bottom-up aspects of active search in a real-world environment. Canadian Journal of Experimental Psychology, 68(1), 8-19.

Hedden, T., \& Zhang, J. (2002). What do you think I think of you? Strategic reasoning in matrix games. Cognition, 85, 1-36.

Hiatt, L. M., \& Trafton, J. G. (2015). Understanding second order theory of mind. In J. A. Adams, W. Smart, B. Mutlu, \& L. Takayam (Eds.), Proceedings of the Tenth Annual ACM/IEEE International Conference on Human-Robot Interaction (pp. 167-168). New York: ACM.

Legge, E. L. G., Spetch, M. L., Cenkner, A., Bulitko, V., Anderson, C., Brown, M., \& Heth, D. (2012). Not all locations are created equal: Exploring how adults hide and search for objects. PLoS ONE, 7, e36993. https://doi.org/10.1371/journal.pone.0036993

Nakayama, K., \& Martini, P. (2011). Situating visual search. Vision Research, 51, 1526-1537. https://doi.org/10.1016/j.visres.2010.09.003

Peskin, J., \& Ardino, V. (2003). Representing the mental world in children's social behavior: Playing hide-and-seek and keeping a secret. Social Development, 12(4), 496-512. https://doi.org/10.1111/14679507.00245

Smilek, D., Weinheimer, L., Kwan, D., Reynolds, M., \& Kingstone, A. (2009). Hiding and finding: The relationship between visual concealment and visual search. Attention, Perception \& Psychophysics, 71(8), 1793-1806. https://doi.org/10.3758/APP. 71.8.1793

Talbot, K. J., Legge, E. L., Bulitko, V., \& Spetch, M. L. (2009). Hiding and searching strategies of adult humans in a virtual and a real-space room. Learning and Motivation, 40(2), 221-233.

Vogeley, K., Bussfeld, P., Newen, A., Herrmann, S., Happé, F., Falkai, P., Maier, W., Shah, N. H., Fink, G. R., \& Zilles, K. (2001). Mind reading: Neural mechanisms of theory of mind and self-perspective. NeuroImage, 14(1), 170-181. https://doi.org/10.1006/nimg.2001.0789

Wolfe, J. M. (2014). Approaches to visual search: Feature integration theory and guided search. In A. C. Nobre \& S. Kastner (Eds.), Oxford Handbook of Attention (pp. 11-15). New York: Oxford University Press. 